\title{
Age and gender changes in children and adolescent patients of a Brazilian eating disorder program
}

\author{
Felipe Alckmin-Carvalho1,2, Alicia Weisz Cobelo2, Márcia Helena da Silva Melo ${ }^{1}$, Rafael Zeni², \\ Vanessa DentZien Pinzon ${ }^{2}$ \\ 1 Instituto de Psicologia da Universidade de São Paulo (IP-USP), São Paulo, SP, Brazil. \\ 2 Instituto de Psiquiatria do Hospital das Clínicas da Faculdade de Medicina da Universidade de São Paulo (IPq-HC-FMUSP), São Paulo, SP, Brazil.
}

Received: $1 / 14 / 2016$ - Accepted: 2/8/2017

DOl: 10.1590/0101-60830000000113

\begin{abstract}
Background: International studies have demonstrated an increase in the prevalence of boys and a decrease of patients' age at the beginning of outpatient treatment for eating disorders (ED). Objective: To evaluate if these changes are also present in the Brazilian population participating in the PROTAD, a Brazilian ED program, and to discuss its clinical implication for treatment. Methods: Cross-sectional study. We evaluated 150 medical records of patients under 18 years diagnosed with ED (DSM IV-TR). Patients were divided into two groups: G1 (2001-2007) ( $\mathrm{n}=77)$ and G2 (2008-2014) ( $\mathrm{n}=73)$. The girl/boy proportion and the mean age of patients were compared. Results: In G1, six boys (7.8\%) were admitted (girl/boy proportion: 11.8:1), while in G2, 16 (22\%) boys were admitted (girl/boy proportion: 3.5:1) $(\mathrm{p}<0.05)$. The mean age in G1 was 15.6 years $(\mathrm{SD}=1.7 ; 95 \% \mathrm{CI}$ : 15.2-15.9), whereas the mean age in G2 was 14.9 years ( $\mathrm{SD}=1.9 ; 95 \% \mathrm{CI}: 14.4-15.3)(\mathrm{p}>0.05)$. Discussion: The increase in the number of boys treated for EDs reported in international studies was also found at the PROTAD. Contrary to what has been reported in international studies, the mean age of patients at the PROTAD did not decrease significantly. Gender and sexual orientation issues, clinical presentation, prior overweight history and culture/media impact on boys should be addressed by the healthcare team to increase the therapeutic efficacy.
\end{abstract}

Alckmin-Carvalho F et al. / Arch Clin Psychiatry. 2017;44(2):33-4

Keywords: Eating disorders, gender, childhood, outpatient treatment, boys.

\section{Introduction}

Eating disorders (EDs) often affect young women ${ }^{1}$. Studies from the 1980s and 1990s have indicated a girl/boy proportion of 1012:1 among patients with ED admitted to outpatient treatment or inpatient unit ${ }^{2}$. However, international studies have shown that the epidemiology of this pathology has changed in the last decades ${ }^{3-5}$.

More recently, studies have found an increase in the frequency of ED among boys ${ }^{3-5}$. A study conducted in Australia with a sample of 101 children and adolescents treated in an ED program found that $25 \%(1: 4)$ of the participants were boys ${ }^{6}$. In terms of the age of patients treated at ED treatment centers, in the United States, a national estimate indicated that, between the years 2005/2006, of the 28,0155 patients, 1,126 (4\%) were children under 12 years old, an increase of $119 \%$ in child admissions when compared to the years 1999/20007. Madden et al. ${ }^{6}$ found that of 79 hospitalized patients, 11 (14\%) were children under 10 years old, whereas of the 22 patients in outpatient treatment, four (18\%) were younger than 10 years old.

Despite the significant number of children and the increased frequency of boys treated at ED programs shown in international studies, there are few studies in Brazil analyzing this trend. The identification of changes in the mean age and gender proportion of patients with ED is important to understand changes in the psychopathology, improve methods of assessment, and provide more focused interventions in this population to increase the effectiveness of the treatment centers. Therefore, the aim of this study was to evaluate if age and gender proportion changes are also present in a Brazilian ED Program, and to discuss its clinical implication for treatment.

\section{Methods}

We conducted a cross-sectional study consisting of the analysis of 150 medical records of patients who sought treatment at the
"Programa de Atendimento, Ensino e Pesquisa em Transtornos Alimentares na Infância e Adolescência" (PROTAD), based on a Brazilian hospital (Institute of Psychiatry, School of Medicine, University of Sao Paulo (IPq-HC-FMUSP). The PROTAD has provided multidisciplinary outpatient and inpatient care for children and adolescents with EDs and their families since November 2001. Its activities are developed at a public institution, and the target population consists of individuals belonging to lower social classes. All the staff members are volunteers. Patients are referred by health care facilities, but mainly, directly via email.

The patients were divided into two groups according to the year of enrollment in the program: G1, from 2001 to 2007, and G2, from 2008 to 2014. The mean age and the number of boys and girls treated in each period were compared. For data collection, we searched sociodemographic questionnaires filled out by the patient's parents/ caregivers upon admission to the outpatient treatment.

Participants under 12 years old were classified as children based on the Brazilian Statute of Children and Adolescents (1990). Student's $t$ test for independent samples was used to compare the mean age. To determine gender proportion, we used the $\mathrm{Z}$ test to investigate the difference between proportions. The statistical analyses were performed using the SPSS software. Patients/caregivers signed an informed consent form. The study was approved by the Research Ethics Committee for studies involving human subjects of the HCFMUSP, under the protocol number 0800/08.

\section{Results and discussion}

Between 2001 and 2014, 167 children and adolescents diagnosed with ED according to the DSM IV-TR criteria were admitted for treatment at the PROTAD ${ }^{8}$. Seventeen $(10.1 \%)$ patients were excluded from the study because they were participating in research projects that actively sought girls or age-specific 
participants. The final sample consisted of 150 patients, 22 boys (14.7\%) and 128 girls (85.3\%) (girl/boy proportion: 6.8:1). G1 was composed of 77 patients, six boys (7.8\%) and 71 girls $(92.2 \%)$ (girl/boy proportion: 11.8:1). G2 consisted of 73 patients, 16 boys (22\%) and 57 girls (78\%) (girl/boy proportion: 3.5:1). There was a statistically significant difference in terms of proportion of boys between $\mathrm{G} 1$ and $\mathrm{G} 2(\mathrm{Z}=-2.44, \mathrm{p}=0.01)$.

Between 2001 and 2014 , the mean age upon admission to treatment $(\mathrm{n}=150)$ was $15.2(\mathrm{SD}=1.8$; 95\%CI: 14.9-15.4). G1's mean age of patients was 15.6 years $(\mathrm{SD}=1.7 ; 95 \% \mathrm{CI}$ : $15.2-15.9)$ and G2's mean age was 14.9 years $(\mathrm{SD}=1.9$; $95 \% \mathrm{CI}$ : $14.4-15.3$ ) $(\mathrm{p}>0.05)$. There were three children (3.8\%) in G1 and seven children $(9.6 \%)$ in $\mathrm{G} 2(\mathrm{p}>0.05)$.

Although there are more girls than boys who are admitted and treated, this difference has been decreasing at the PROTAD. The frequency of boys in G2 is similar to the one found by Madden et al. ${ }^{6}(25 \%)$, and much higher than those reported by studies from the 1990 s, showing a proportion of 10-12:12.

It is worth highlighting the implications of the increase in the number of boys admitted to outpatient treatment at younger ages, as boys with ED have specific characteristics and needs $s^{3,4,9-11}$. Strother et al. ${ }^{1}$ pointed out that health professionals should be aware of the differences in boys' weight history, once a history of overweight or prior obesity is more frequent among male patients when compared to girls. A prior history of overweight or obesity may intensify the fear of gaining weight and impair adherence to treatment. It is useful to ensure patients that after breaking the binge/purge/restrict cycle, eating in quantity compatible with their weight, age, and metabolism will not make them obese.

As a method to control weight, male teenagers and young adults tend to exercise in excess, which is associated with the use of steroids and/or growth hormone. This aspect differs substantially from what have been observed among girls, who resort more frequently to induce vomiting and fasting periods, and therefore it should be considered when evaluating boys with $\mathrm{ED}^{1,5}$.

Clinicians observe that, especially in cases of anorexia nervosa among boys, ED can be expressed as an avoidance response to difficulties related to acceptance of sexual orientation. In such cases, severe malnutrition conditions produce decreasing testosterone levels, resulting in decreased libido and less contact with sexuality issues ${ }^{1}$. Thus, gaining weight during treatment brings back these difficulties that can hamper recovery and must be concomitantly addressed in psychotherapy.

Analyses of advertising campaigns of products developed for men over recent decades have shown more muscular bodies and, at the same time, less body fat percentage. It is important to be attentive, as developed muscles can hide an ED, giving a false idea of health and hindering diagnosis and treatment ${ }^{1,10}$.

Our descriptive analysis showed a slight decrease in the mean age of G2 compared to the global mean and to G1. The number of children in G2 is larger than that found in G1 and close to the $4 \%$ reported by Zhao and Encinosa ${ }^{7}$. Early age at admission may be associated with earlier onset of the disease and/or early recognition of signs and symptoms by caregivers and health professionals.

Researchers have pointed out that the clinical presentation of children with ED differs substantially from that of adolescent and adult patients in the following aspects: shorter disease duration, fewer symptoms of ED, lower frequency of binge/purge and physical exercises to control weight, faster loss of weight, and increased risk of growth problems ${ }^{9,11}$.

In terms of primary prevention, it is necessary to investigate how obesity prevention campaigns based on fatphobia and stigmatization, as well as the media, influence boys to seek muscular and slim bodies, and what is its relationship with the increased number of ED cases among boys. Further studies are necessary to adapt the assessment and diagnosis methods for boys, and to test the effectiveness of treatment offered to the male population, covering specific issues, such as those addressed in this study.

Our results are unparalleled in Brazil, where there are very few studies on ED during childhood or adolescence. Such results help improve our knowledge in this field by demonstrating changes in the profile of outpatients and by discussing the impact of such changes on the treatment. However, such results must be analyzed with caution due to some limitations of the study. In spite of the statistically significant difference between the proportion of boys in G1 and G2, the reduced absolute number of boys in the sample (six and 16), as well as the exclusion of a significant proportion of the sample, initially (10.1\%) imply a low statistical power that impairs the internal and external validity of the study. Although most families of patients with ED contact PROTAD directly by email, the increased number of boys in treatment may reflect more severe cases at a tertiary center of care and not necessarily an epidemiologic change of ED.

The changes found in international studies were also found in the population treated at the PROTAD, although only the difference in the girl/boy proportion showed statistically significant difference. Gaining knowledge about the specific characteristics and needs of children and adolescents with ED helps improve specialized public health services. By focusing on the specific needs of this population, we can increase treatment adherence and promote the effectiveness of the interventions.

\section{References}

1. Strother E, Lemberg R, Stanford SC, Turberville D. Eating disorders in men: underdiagnosed, undertreated, and misunderstood. Eat Disord. 2012;20(5):346-55.

2. Nielsen S. The epidemiology of anorexia nervosa in Denmark from 1973 to 1987: a nationwide register study of psychiatric admission. Acta Psychiatr Scand. 1990;81(6):507-14

3. Bryant-Waugh R. Feeding and eating disorders in children. Curr Opin Psychiatry. 2013;26(6):537-42.

4. Campbell K, Peebles R. Eating disorders in children and adolescents: state of the art review. Pediatrics. 2014;134(3):582-92.

5. Rosen DS. Identification and management of eating disorders in children and adolescents. Pediatrics. 2010;126(6):1240-53.

6. Madden S, Morris A, Zurynski YA, Kohn M, Elliot EJ. Burden of eating disorders in 5-13-year-old children in Australia. Med J Aust. 2009;190(8):410-4.

7. Zhao Y, Encinosa W. Hospitalizations for eating disorders from 1999 to 2006. HCUP statistical brief \#70. 2009. Available from: <http://www. hcup-us.ahrq.gov/reports/statbriefs/sb70.pdf $>$. Accessed on: Nov. 26, 2015.

8. American Psychiatric Association. Diagnostic and Statistical Manual of Mental Disorders (DSM-IV-TR). Washington: PA; 2003.

9. Peebles R, Wilson JL, Lock JD. How do children with eating disorders differ from adolescents with eating disorders at initial evaluation? J Adolesc Health. 2006;39(6):800-5.

10. Räisänen $U$, Hunt $K$. The role of gendered constructions of eating disorders in delayed help-seeking in men: a qualitative interview study. BMJ Open. 2014;4(4):e004342.

11. Walker T, Watson HJ, Leach DJ, McCormack J, Tobias K, Hamilton MJ, et al. Comparative study of children and adolescents referred for eating disorder treatment at a specialist tertiary setting. Int J Eat Disord. 2014;47(1):47-53. 